\title{
The Effects of Nonnutritive Sweeteners on the Cariogenic Potential of Oral Microbiome
}

\author{
Jianhui Zhu $\left(\mathbb{D},{ }^{1,2}\right.$ Jiaxin Liu $\left(\mathbb{D},{ }^{1,2}\right.$ Zhengyi Li $\mathbb{D},{ }^{1}$ Ranhui Xi $\mathbb{D}^{1,2}$ Yuqing Li $\mathbb{D}^{1},{ }^{1}$ Xian Peng $(\mathbb{D})$, \\ Xin Xu $\mathbb{D}^{1,2}$ Xin Zheng $\mathbb{D}^{1,2}$ and Xuedong Zhou $\mathbb{D}^{1,2}$ \\ ${ }^{1}$ State Key Laboratory of Oral Diseases \& National Clinical Research Center for Oral Diseases, West China Hospital of Stomatology, \\ Sichuan University, Chengdu 610041, China \\ ${ }^{2}$ Department of Cariology and Endodontics, West China Hospital of Stomatology, Sichuan University, Chengdu 610041, China
}

Correspondence should be addressed to Xin Zheng; zxzdg@126.com and Xuedong Zhou; zhouxd@scu.edu.cn

Received 14 March 2021; Revised 7 June 2021; Accepted 18 June 2021; Published 25 June 2021

Academic Editor: Maria Bayliak

Copyright (c) 2021 Jianhui Zhu et al. This is an open access article distributed under the Creative Commons Attribution License, which permits unrestricted use, distribution, and reproduction in any medium, provided the original work is properly cited.

\begin{abstract}
Nonnutritive sweeteners (NNSs) are sugar substitutes widely used to reduce the negative health effects of excessive sugar consumption. Dental caries, one of the most prevalent chronic diseases globally, results from a pathogenic biofilm with microecological imbalance and frequent exposure to sugars. Some research has shown that certain NNSs possess less cariogenic potential than sucrose, indicating their putative effect on oral microbiome. To uncover the alterations of acidogenic pathogens and alkali-generating commensals, as well as the biofilm cariogenic potential under the influence of NNSs, we selected four common NNSs (acesulfame-K, aspartame, saccharin, and sucralose) and established single-, dual-, and multispecies in vitro culture model to assess their effects on Streptococcus mutans (S. mutans) and/or Streptococcus sanguinis (S. sanguinis) compared to sucrose with the same sweetness. The results showed that NNSs significantly suppressed the planktonic growth, acid production, and biofilm formation of $S$. mutans or S. sanguinis compared with sucrose in single-species cultures. Additionally, decreased S. mutans/S. sanguinis ratio, less EPS generation, and higher $\mathrm{pH}$ value were observed in dual-species and salivaderived multispecies biofilms with supplementary NNSs. Collectively, this study demonstrates that NNSs inhibit the cariogenic potential of biofilms by maintaining microbial equilibrium, thus having a promising prospect as anticaries agents.
\end{abstract}

\section{Introduction}

Sugar is the generic name for sweet-tasting, soluble carbohydrates. For most people, "sugar" refers to the granulated household flavoring to enhance sweetness, whose main ingredient is sucrose, one of the most commonly consumed sugars in human diet [1]. Although sugar maintains an important position in food industry, its excessive consumption has been implicated with several health conditions including obesity, diabetes, cardiovascular diseases, and dental caries [2]. Due to public health efforts to lower sugar intake, the consumption of alternative sweeteners has been increasing globally [3].

Dental caries is one of the most prevalent chronic diseases worldwide, characterized by local destruction of dental hard tissues. It is a multifactorial disease that occurs in the presence of a pathogenic biofilm and frequent exposure to sugars [4]. Considered as a highly cariogenic substrate, sucrose can be utilized by pathogenic bacteria to generate acid by-product which ultimately leads to demineralization, as well as extracellular polysaccharides (EPS) which facilitate its colonization and form a protective environment against host defense [5]. From an ecological perspective, the interactions between acidogenic/aciduric bacteria (e.g., Streptococcus mutans and Lactobacillus spp.) and alkali-generating commensals (e.g., Streptococcus sanguinis and Streptococcus gordonii) in dental plaque are closely associated with the initiation and development of caries $[6,7]$.

Sugar substitutes have been identified to contribute in caries prevention, as many of them cannot be utilized efficiently by oral bacteria to produce acids [8]. Certain sugar substitutes have been reported to possess less cariogenic potential than sucrose in in vitro biofilm models established on glass beads or enamel slabs, with one or more bacteria 
strains inoculated [9-11]. However, their effects on the antagonism between acidogenic and alkali-generating bacteria based on saliva-derived multispecies biofilm model have not yet been investigated. Alternative sweeteners can be categorized into nutritive sweeteners (also called bulk or caloric sweeteners) and nonnutritive sweeteners (also called intense or noncaloric sweeteners) according to their different ability to provide energy. Nonnutritive sweeteners (NNSs) are many times sweeter than equally weighted sugar while contributing few or no calories, and are commonly used in a wide range of foods [12].

This study is aimed to assess the effect of four NNSs (acesulfame-K, aspartame, saccharin, and sucralose) on the cariogenic potential of biofilms, which are extensively applied and considered safe for consumption by the United States Food and Drug Administration (FDA) [13]. We measured the growth, biofilm formation, and acid/EPS production of S. mutans and S. sanguinis after they were cultured alone or in dual- and multispecies biofilm models and comparatively evaluated the results of NNSs groups with reference to sucrose of the same sweetness.

\section{Materials and Methods}

2.1. Materials and Reagents. Brain Heart Infusion (BHI) was purchased from BD Difco (USA). Acesulfame-K, crystal violet $(\mathrm{CV})$, Tris hydrochloride (Tris- $\mathrm{HCl}$ ), Ethylenediaminetetraacetic acid (EDTA), and lysozyme were purchased from Sigma (USA). Saccharin, sucralose, sodium dodecyl sulfate (SDS), and formamide were purchased from J\&K scientific (China). Aspartame was purchased from APExBIO (USA). Alexa Fluor 647-labeled dextran conjugate, SYTO 9 green fluorescent nucleic acid stain $(485 / 498 \mathrm{~nm})$, and phosphatebuffered saline (PBS, $50 \mathrm{mM}, \mathrm{pH}$ 6.8) were purchased from Thermo Fisher Scientific (USA).

2.2. Bacterial Culture. S. mutans UA159 and S. sanguinis ATCC10556 were commercially obtained from the American Type Culture Collection (ATCC). S. mutans and S. sanguinis were grown at $37^{\circ} \mathrm{C}$ and $5 \% \mathrm{CO}_{2}$ in $\mathrm{BHI}$ medium. They were cultured overnight and adjusted to optical density (OD) 0.5 0.6 at $600 \mathrm{~nm}\left(1 \times 10^{8} \mathrm{CFU} / \mathrm{ml}\right.$ based on the $\mathrm{OD}_{600 \mathrm{~nm}}$ versus $\mathrm{CFU} / \mathrm{ml}$ curve of each strain) and further $1: 100$ diluted in the growth medium for inoculation.

2.3. Preparation of Different Growth Media. The liquid medium containing one of the NNSs (acesulfame-K, saccharin, aspartame, or sucralose) was prepared to a concentration which has the same sweetness degree as sucrose according to information published by FDA (Table 1). For planktonic growth or single-species biofilm, BHI without any added sweeteners was considered as the negative control, and $\mathrm{BHI}$ with $1 \%$ sucrose was considered as the positive control. In the study of dual- and multispecies biofilm, the prepared media for all groups were supplemented with $0.2 \%$ sucrose to ensure sufficient biofilm adhesion for further analyses. For instance, the medium of acesulfame- $K$ group contained $0.2 \%$ sucrose and acesulfame- $\mathrm{K}$ maintaining sweetness comparable with $0.8 \%$ sucrose. BHI with $0.2 \%$ sucrose was con- sidered as the negative control, and BHI with $1 \%$ sucrose was considered as the positive control. All of the media were filter-sterilized and stored at $4^{\circ} \mathrm{C}$ before use.

2.4. Planktonic Growth of S. mutans and S. sanguinis. Overnight cultures of $S$. mutans and $S$. sanguinis were inoculated separately in six sterile $15 \mathrm{ml}$ centrifuge tubes, each of them containing $5 \mathrm{ml}$ different growth media (BHI, BHI with $1 \%$ sucrose, and $\mathrm{BHI}$ with four sweeteners). After incubation at $37^{\circ} \mathrm{C}$ and $5 \% \mathrm{CO}_{2}$ for $24 \mathrm{~h}$, we suspended the cultures and measured their $\mathrm{OD}_{600 \mathrm{~nm}}$ using Nanodrop One (Thermo Fisher Scientific Waltham, MA, USA) with cuvettes (light path $=10 \mathrm{~mm}$ ). The cultures were then recollected, centrifuged at $5000 \mathrm{~g}$ for $10 \mathrm{~min}$ to remove the precipitation. The $\mathrm{pH}$ values of culture supernatants were then recorded by $\mathrm{pH}$ electrode InLab Expert Pro (Mettler Toledo, Melbourne, Australia).

2.5. Crystal Violet Staining of Single-Species Biofilm. The biofilm formation of $S$. mutans or $S$. sanguinis grown in media containing different NNSs was observed after CV staining. Overnight cultures of each strain were inoculated in a 96well microtiter plate, each well containing $200 \mu \mathrm{l}$ medium (BHI, BHI with $1 \%$ sucrose, and BHI with four sweeteners). After cultivation for $24 \mathrm{~h}$, we aspirated the culture supernatant, washed each well three times with PBS, fixed the biofilms with methanol for $15 \mathrm{~min}$, and stained with $0.1 \%(w / v) \mathrm{CV}$ for 10 min. The redundant CV was removed by PBS rinses until the rinse solution appeared colorless. The images of the wells were captured by a stereo microscope (Olympus SZX10, Japan). The biofilms were then destained by adding $200 \mu \mathrm{l}$ $33 \%$ acetic acid to each well and shaking the plate at $37^{\circ} \mathrm{C}$ (100 r/min) for $30 \mathrm{~min}$. The destaining solution of each well was added to another blank 96-well microtiter plate before the $\mathrm{OD}_{595 \mathrm{~nm}}$ value was measured. The experiment was carried out with triplicate samples and replicated three times, with data normalized by the blank wells.

2.6. Dual- and Multispecies Biofilm Model. Dual-species biofilm model was established as described previously [14]. Whole unstimulated saliva was collected on ice from 5 healthy adult volunteers who were asked not to eat or drink $2 \mathrm{~h}$ before collection. The saliva was pooled and clarified by low-speed certification ( $2600 \mathrm{~g}$ for $10 \mathrm{~min}$ ). The supernatant was filtered for sterilization and stored at $-80^{\circ} \mathrm{C}$ before use. A 24-well plate with a glass coverslip in each well was used for biofilm culture. We added $500 \mu \mathrm{l}$ sterile saliva to each well and incubated the plate at $37^{\circ} \mathrm{C}$ for $2 \mathrm{~h}$ to coat the glass coverslips. After the saliva was aspirated, the growth medium was added into each well. (BHI with $0.2 \%$ sucrose, BHI with $1 \%$ sucrose, and BHI with four sweeteners). Overnight cultures of $S$. mutans and $S$. sanguinis were inoculated on saliva-coated glass coverslips (inoculum ratio $=1: 1$ ).

Saliva-derived multispecies biofilm model was established according to Guo et al. [15] with modifications. Whole unstimulated saliva was collected on ice from 5 healthy adult volunteers without antibiotic treatment in the last 3 months or any diseases that might influence the saliva composition. Each sample was centrifuged at $2600 \mathrm{~g}$ for $10 \mathrm{~min}$ and pooled 
TABLE 1: Concentrations of sucrose and NNSs in growth media.

\begin{tabular}{lcccc}
\hline Sweeteners & Relative sweetness & Molar mass $(\mathrm{g} / \mathrm{Mol})$ & Concentration $(\mathrm{g} / 100 \mathrm{ml})$ & Concentration $(\mathrm{Mol} / \mathrm{L})$ \\
\hline Sucrose & 1 & 342.3 & 1 & $2.92 \mathrm{E}-02$ \\
Acesulfame-K & 200 & 201.24 & $5.00 \mathrm{E}-03$ & $2.48 \mathrm{E}-04$ \\
Aspartame & 200 & 294.3 & $5.00 \mathrm{E}-03$ & $1.70 \mathrm{E}-04$ \\
Saccharin & 300 & 183.18 & $3.33 \mathrm{E}-03$ & $1.82 \mathrm{E}-04$ \\
Sucralose & 600 & 397.64 & $1.67 \mathrm{E}-03$ & $4.19 \mathrm{E}-05$ \\
\hline
\end{tabular}

together. To collect a stock of the saliva-derived multispecies bacteria, $1 \mathrm{ml}$ pooled saliva was added into $5 \mathrm{ml} \mathrm{BHI}$ and incubated under anaerobic conditions at $37^{\circ} \mathrm{C}$ for $24 \mathrm{~h}$. The cultures were centrifuged at $14000 \mathrm{~g}$ for $3 \mathrm{~min}$, resuspended in fresh BHI with $25 \%$ glycerol, and kept at $-80^{\circ} \mathrm{C}$. Overnight cultures of $S$. mutans and the saliva-derived multispecies bacteria were resuspended into $\mathrm{BHI}$ to $\mathrm{OD}_{600 \mathrm{~nm}}$ of $0.5-0.6$, respectively, and mixed in a $1: 10$ volume ratio. The mixtures were then $1: 100$ diluted in fresh BHI and added to a 24-well plate with sterile saliva-coated glass coverslips.

Both dual- and multispecies biofilm samples were grown at $37^{\circ} \mathrm{C}$ for $24 \mathrm{~h}$ before further analyses. The culture medium was collected to measure $\mathrm{pH}$ values after $24 \mathrm{~h}$ cultivation.

2.7. Bacteria/Extracellular Polysaccharides Staining. The bacteria and their EPS production were determined by bacteria/EPS staining as described previously [16]. Briefly, $1 \mu \mathrm{M}$ Alexa Fluor 647-labeled dextran conjugate was added to the culture medium at the beginning of incubation. The labeled dextran serves as a primer for Glucosyltransferases and can be simultaneously incorporated during EPS synthesis in biofilm development [17]. The bacterial cells were labeled with 2 $\mu \mathrm{M}$ SYTO 9 green fluorescent nucleic acid stain (485/498 $\mathrm{nm}$ ) through $15 \mathrm{~min}$ staining after cultivation. The imaging was performed using a confocal laser scanning microscope (Olympus FV3000, Japan) equipped with a 60x (1.42 numerical aperture) oil immersion objective lens. The scan speed, photomultiplier detector gain, and pinhole aperture were kept constant for all image stacks. Representative images were demonstrated using Imaris software (v7.0). The quantities of bacteria and EPS were based on 5 image stacks in each group obtained from three independent tests and measured with COMSTAT2 image analysis program [18] as a plugin of Image J software (v1.8) by calculating biomass.

2.8. Fluorescence In Situ Hybridization. The sequences of oligonucleotide probes in fluorescence in situ hybridization (FISH) were either designed using ARB software packages [19] or acquired from previous studies [16]. Universal bacteria probe $\left(5^{\prime}\right.$-GCTGCCTCCCGTAGGAGT- $\left.3^{\prime}\right)$, S. mutans specific probe $\left(5^{\prime}\right.$-ACTCCAGACTTTCCTGAC- $\left.3^{\prime}\right)$, and $S$. sanguinis specific probe $\left(5^{\prime}\right.$-GCATACTATGGTTAAGCCA CAGCC- $3^{\prime}$ ) were labeled with Alexa Fluor 405, Alexa Fluor 488, or Alexa Fluor 594, respectively. The biofilms on glass coverslips were fixed in $4 \%$ paraformaldehyde for $6 \mathrm{~h}$, rinsed with PBS, and dried. For probe penetration, the biofilm samples were treated with $500 \mu \mathrm{l}$ lysis buffer $(100 \mathrm{mM}$ Tris- $\mathrm{HCl}$, $50 \mathrm{mM}$ EDTA, $30 \mathrm{mg} / \mathrm{ml}$ lysozyme, $\mathrm{pH} 8.0$ ) at $37^{\circ} \mathrm{C}$ for 20 min. The biofilms were then rinsed, serially dehydrated in ethanol (50\%, 80\%, and 100\%; 3 min each), and dried. For probe hybridization, each bead was covered with $50 \mu \mathrm{l}$ of hybridization buffer $(0.9 \mathrm{M} \mathrm{NaCl}, 20 \mathrm{mM}$ Tris- $\mathrm{HCl}, 0.01 \%$ SDS, $20 \%$ formamide) containing 3 oligonucleotide probes $\left(2 \mu \mathrm{M}\right.$ each) and incubated at $46^{\circ} \mathrm{C}$ for $90 \mathrm{~min}$ in a closed cassette with a piece of paper towel soaked by hybridization buffer to maintain humidity. Subsequently, the beads were washed with buffer $(20 \mathrm{mM}$ Tris/ $\mathrm{HCl}, 5 \mathrm{mM}$ EDTA, $0.01 \%$ SDS, $215 \mathrm{mM} \mathrm{NaCl}$ ), incubated in the washing buffer at $46^{\circ} \mathrm{C}$ for $15 \mathrm{~min}$, and rinsed in $4^{\circ} \mathrm{C}$ nuclease-free water. The images were captured and analyzed with similar tools in the EPS staining experiment.

2.9. Statistics. The statistical analysis and graphing of the data were performed using the GraphPad Prism software (v8.0), with all data demonstrated as mean \pm standard deviations (SD). Group mean values were compared by one-way ANOVA and Tukey post hoc test. A two-tailed $P<0.05$ was considered to be statistically significant.

\section{Results}

3.1. NNSs Suppress the Growth and Acid Production of S. mutans or S. sanguinis in Planktonic Cultures and SingleSpecies Biofilm. After $24 \mathrm{~h}$ incubation, suspended S. mutans cultures containing NNSs exhibited a significantly lower level of $\mathrm{OD}_{600 \mathrm{~nm}}$ and a significantly higher level of $\mathrm{pH}$ value than that containing 1\% sucrose (Figure 1(a)). Similar significant relatively inhibitory effects were observed against $S$. sanguinis planktonic growth and acid production, but to a lesser extent (Figure 1(b)). Notably, these effects in planktonic bacterial cultures displayed no significant difference between each NNS group. Moreover, CV staining demonstrated the decreased biofilm formation of $S$. mutans or S. sanguinis cultured with NNSs compared to that with sucrose and the decline of $S$. sanguinis biofilms was more obvious than that of S. mutans (Figures 1(c) and 1(d)). The biofilm formed in the sucralose group was significantly increased compared with the negative control while far less than the sucrose group (Figure 1(d)).

3.2. NNSs Diminish S. mutans/S. sanguinis Ratio and Acid/EPS Generation in Dual-Species Biofilms. The influence of NNSs on S. mutans and S. sanguinis was further investigated in dual-species biofilms using FISH and EPS staining. The images of biofilms labeled with species-specific FISH probes showed that NNSs significantly suppressed the absolute biomass of $S$. mutans and the S. mutans/S. sanguinis ratio compared with the $0.2 \%$ sucrose treated control (Figures 2(a) 


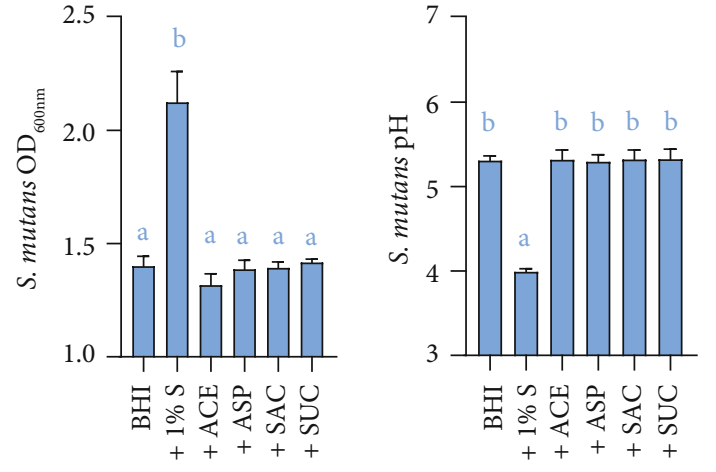

(a)
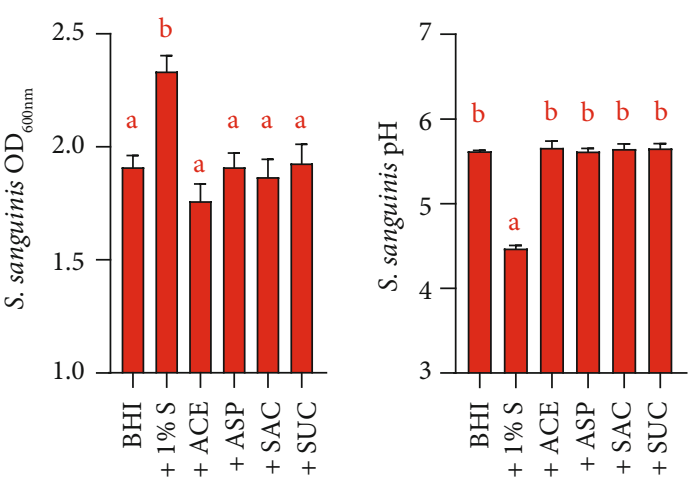

(b)

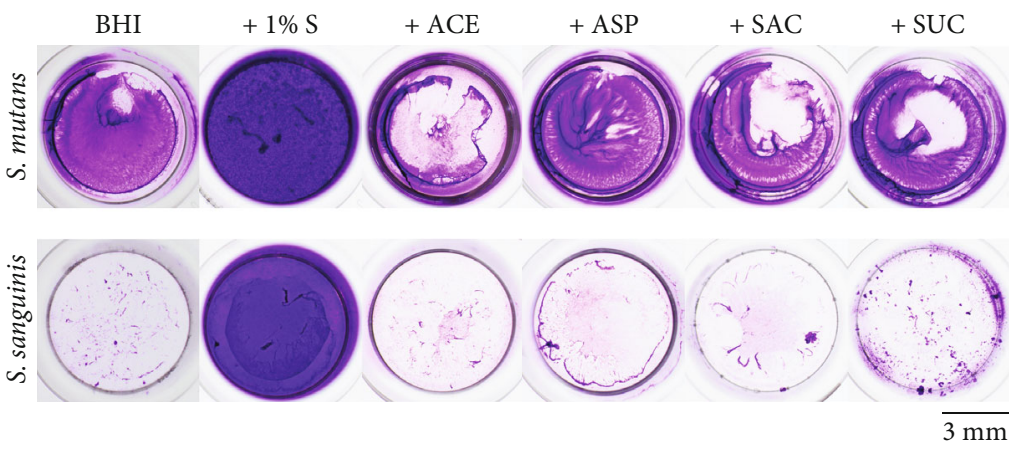

(c)

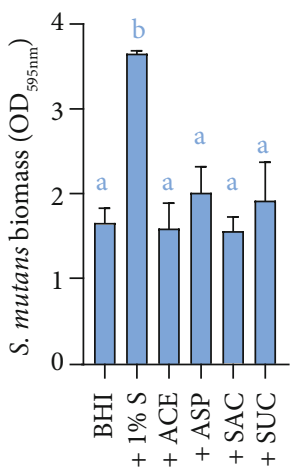

(d)

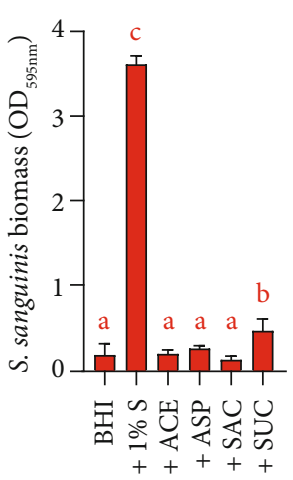

FIGURE 1: Effects of NNSs on the planktonic growth, acid production, and biofilm formation of S. mutans or S. sanguinis. OD $600 \mathrm{~nm}$ and pH value measurement of $S$. mutans (a) and S. sanguinis (b) planktonic cultures, respectively. (c) Representative images of $S$. mutans and $S$. sanguinis biofilms after crystal violet staining. (d) The quantitative data of single-species biofilm biomass after destaining. Results are presented as mean \pm standard deviation. Different lowercase letters indicate a significant intergroup difference and are marked according to mean value. (S: sucrose; ACE: acesulfame-K; ASP: aspartame; SAC: saccharin; SUC: sucralose.).

and 2(b)). However, among four NNSs, only the acesulfame-K group exhibited a significant decrease of absolute $S$. sanguinis biomass, although to a lesser degree than $S$. mutans. It is indicated that NNSs selectively constrained the growth of $S$. mutans while keeping the growth of $S$. sanguinis, thus promoting the relative dominance of $S$. sanguinis in dual-species biofilms. Moreover, significant inhibitory effects on EPS production and higher $\mathrm{pH}$ value of the culture media were observed in dual-species biofilms with NNSs (Figures 2(c)-2(e)).

\subsection{NNSs Reduce Acid/EPS Production of Saliva-Derived} Multispecies Biofilm and Acesulfame-K Decrease $S$. mutans/S. sanguinis ratio. We further examined the effect of NNSs on the microbial composition and EPS/acid production of the multispecies biofilms which were derived from saliva and supplemented with $S$. mutans inoculation. Notably, the $S$. mutans/universal bacteria ratio and $S$. mutans/S. sanguinis ratio were significantly lower in biofilms with acesulfame- $\mathrm{K}$ than other treated groups (even the $0.2 \%$ sucrose group). However, analysis of FISH-labeled multispecies biofilms showed no significant difference among all groups in the biomass ratio of $S$. sanguinis to universal bacteria (Figures 3(a) and 3(b)). These results suggested the particular inhibitory effect of acesulfame-K on typical acidogenic bacteria in a multispecies biofilm. In addition, biofilms devel- oped in media with NNSs exhibited less EPS and acid production compared with $1 \%$ sucrose (Figures 3(c)-3(e)).

\section{Discussion}

Dental caries results from polymicrobial infection in which imbalance between acidogenic pathogens and alkaligenerating commensals in plaque biofilm plays a crucial role $[6,20,21]$. The acid accumulation and $\mathrm{pH}$ decline of microbial biofilm led by its frequent exposure to carbohydrates selectively enrich the acidogenic/aciduric species (e.g., $S$. mutans and lactobacilli) and suppress the less aciduric commensal residents (e.g., S. sanguinis), driving the shift of microbial community to a more cariogenic consortium consequently. This positive feedback leads to a continuous local $\mathrm{pH}$ decline below the critical value which results in the demineralization of tooth tissues and eventually the development of caries lesion $[6,22]$. Sugar substitutes are generally considered to have negative effects on caries initiation [1, 23]. Some research has reported certain sweeteners prominently inhibited the capability of $S$. mutans for biofilm formation and enamel demineralization based on carious lesion model in vitro $[10,11]$. In addition, some sweeteners have shown a decrease in bacterial adherence and biofilm mass production compared with sucrose based on a mixed inoculation of S. mutans, S. sanguinis, and S. mitis on glass 


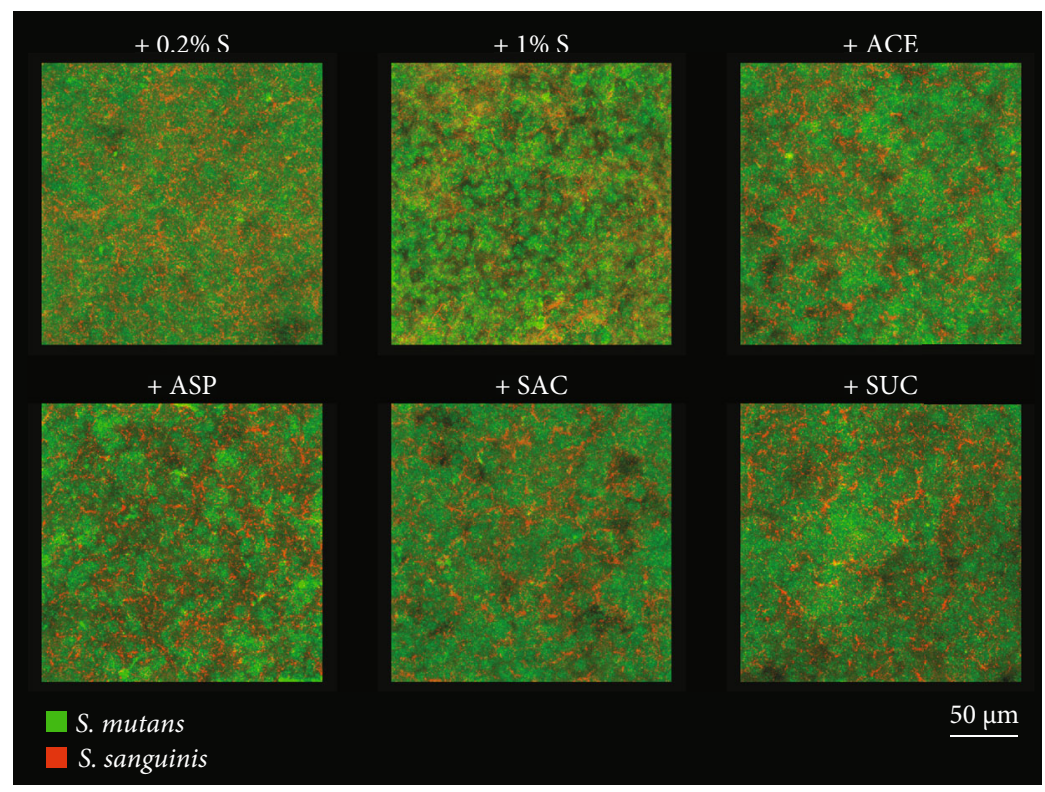

(a)
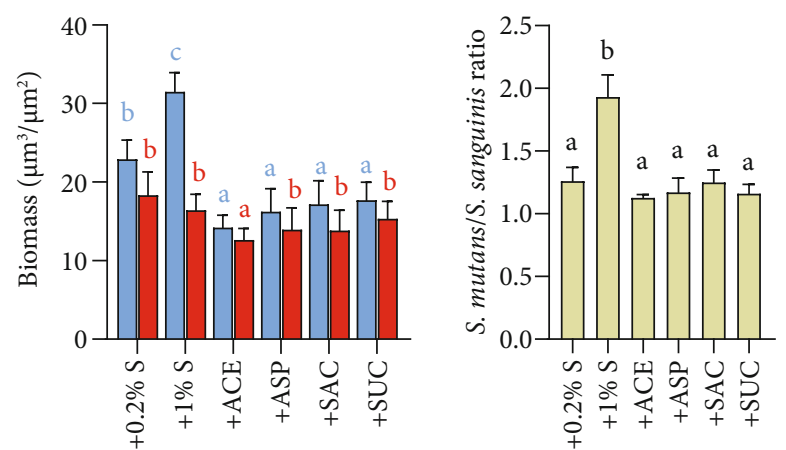

$\square$ S. mutans

S. sanguinis

(b)
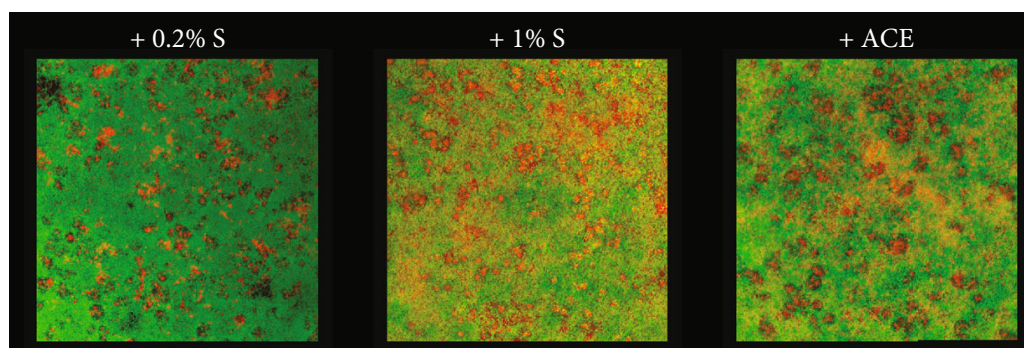

+ ASP

+ SAC

+ SUC
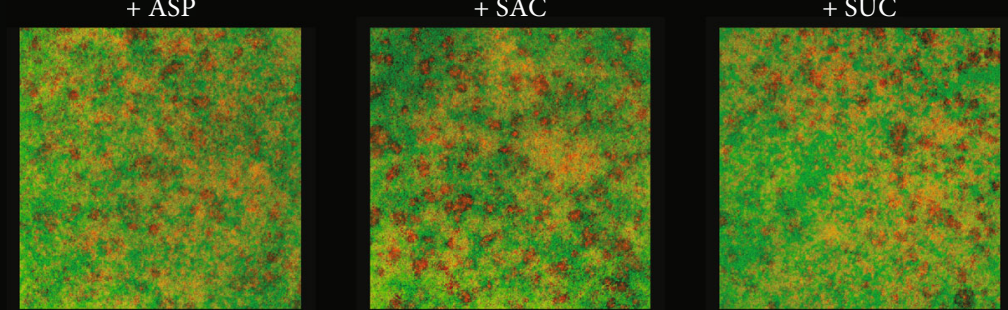

$\square$ Bacteria

$\underline{50 \mu \mathrm{m}}$

$\square$ EPS

(c)

Figure 2: Continued. 


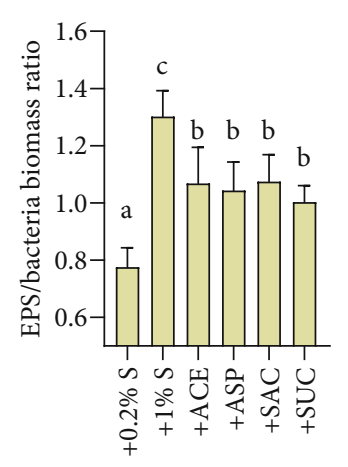

(d)

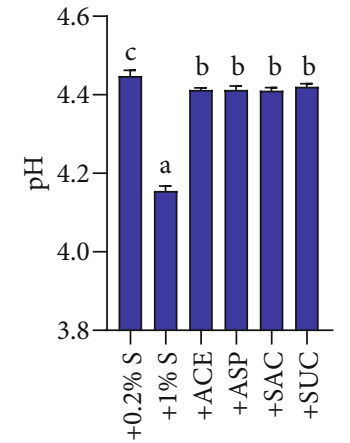

(e)

FIGURE 2: Effects of NNSs on bacterial composition, extracellular polysaccharides (EPS) generation, and acid production of dual-species biofilms. (a) Representative images of dual-species biofilms after fluorescence in situ hybridization. Green, S. mutans; Red, S. sanguinis. (b) Quantitative data of S. mutans/S. sanguinis biomass and bacterial composition. (c) Representative images of S. mutans/S. sanguinis dualspecies biofilms after EPS staining. Green, bacteria; red, EPS. (d) The quantitative data of EPS/bacterial amount in dual-species biofilms. (e) The $\mathrm{pH}$ values of culture media of dual-species biofilms. Results are presented as mean \pm standard deviation. Different lowercase letters indicate a significant intergroup difference and are marked according to mean value. (S: sucrose; ACE: acesulfame-K; ASP: aspartame; SAC: saccharin; SUC: sucralose.).

beads [9]. However, it is still unknown how sugar substitutes influence the cariogenic potential of oral biofilms closer to the real situation, as well as the competitive advantage between acidogenic pathogens and commensal bacteria. The present study has for the first time, uncovered the effect of NNSs on biofilm formation and its EPS/acid production from an ecological perspective in dual-species and salivaderived multispecies models.

Initially, we observed that on pure cultivation, NNSs not merely prohibited the growth and acid production of $S$. mutans, but also that of $S$. sanguinis, indicating the necessity to investigate the influence of NNSs on interactions between different species and the collective cariogenic potential of oral biofilms. Since the phenomenon of antagonism between $S$. mutans and S. sanguinis is a typical in vitro microcosm of interactions between acidogenic and alkali-generating bacteria $[24,25]$, we employed the commonly utilized dual-species model comprised of S. mutans and S. sanguinis to study bacteria interplay. The results indicated that the metabolism of dual-species biofilm to produce acids was active in high sucrose condition, and the aciduric $S$. mutans made competitive predominance over $S$. sanguinis (with their biomass ratio close to 2). Additionally, the $\mathrm{pH}$ value of dual-species biofilms with NNSs was higher than that of sucrose, and the S. mutans/S. sanguinis ratio significantly declined to about 1 , suggesting the inhibitory effects of NNSs on biofilm acid production and $S$. mutans advantage. These outcomes demonstrated the ecological benefit of NNSs on the perspective of the in vitro competition between acidogenic and alkali-generating bacteria.

To further identify the influence of NNSs on overall biofilm cariogenic potential, we established the saliva-derived multispecies biofilm model. Acid production, acidogenic/alkali-generating bacteria biomass ratio, and EPS generation are critical evaluation indicators for the cariogenic ability of biofilms, and each of them has a close association with others. $S$. mutans and other acidogenic organisms can metabolize fermentable sugars to produce acids which are responsible for cariogenesis $[26,27]$. EPS generated by exoenzymes from $S$. mutans are considered as essential virulence factors of dental caries, as they form the core of the scaffold of cariogenic biofilms, provide abundant primary binding sites, and enhance bacteria cohesion [5]. EPS and other materials in the matrix could restrict the capability of saliva for neutralizing acids, contributing to the formation and maintenance of acidic microenvironments [28]. The acid microenvironment may induce decreased abundance of $S$. sanguinis which is more sensitive to acidic condition [29]. In contrast, as the most prevalent species contains the arginine deiminase system, $S$. sanguinis is capable of producing alkali to gain a competitive advantage over $S$. mutans, thus preserving $\mathrm{pH}$ homeostasis and protecting against caries [30,31]. This study revealed that NNSs could significantly reduce the biofilm formation, EPS production, and S. mutans/S. sanguinis ratio, as well as increasing biofilm $\mathrm{pH}$. Although the decline of $S$. mutans/S. sanguinis ratio primarily exhibited as an inclination (only acesulfame-K group significantly distinguished from sucrose group), the results of our experiments collectively provided elementary evidence for NNSs' effect of diminishing biofilm cariogenic capacity.

It is worth noting that there were some differences in the results of NNSs' effect on bacterial growth between the single-species biofilm model and the multispecies biofilm model. When cultured separately with NNSs, the biomass of S. mutans biofilms was higher than that of S. sanguinis, while coculture and culture with saliva-derived bacteria did not show obviously increased growth of $S$. mutans relative to $S$. sanguinis. We speculate one of the reasons is that the stronger capacity for EPS production of $S$. mutans made the single-species biofilms more adhesive against PBS rinses to remove the excess $\mathrm{CV}$. Moreover, since the interaction between different bacteria strains may influence the ultimate effect of external substances, it is necessary to apply the biofilm model comprised of a more complex microbial community. However, it is still important to highlight that the results of the present study were attained by in vitro approach. 


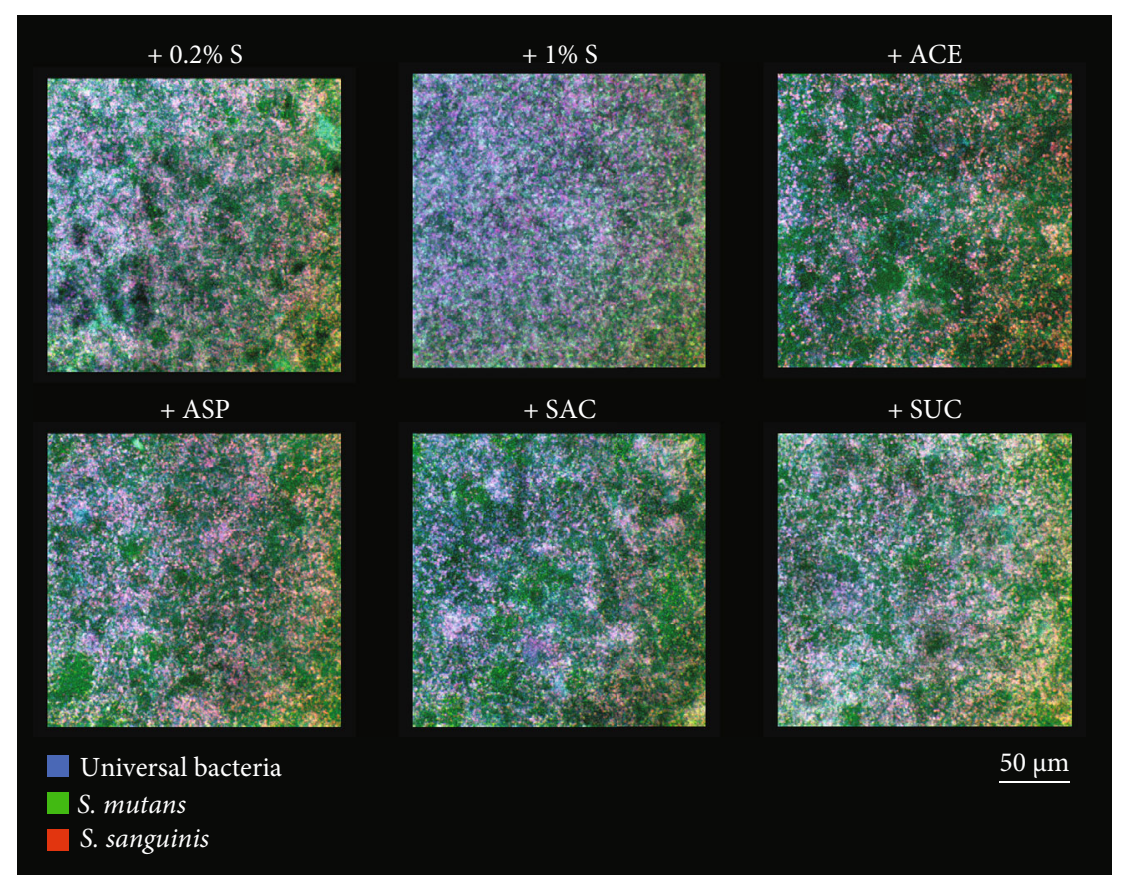

(a)
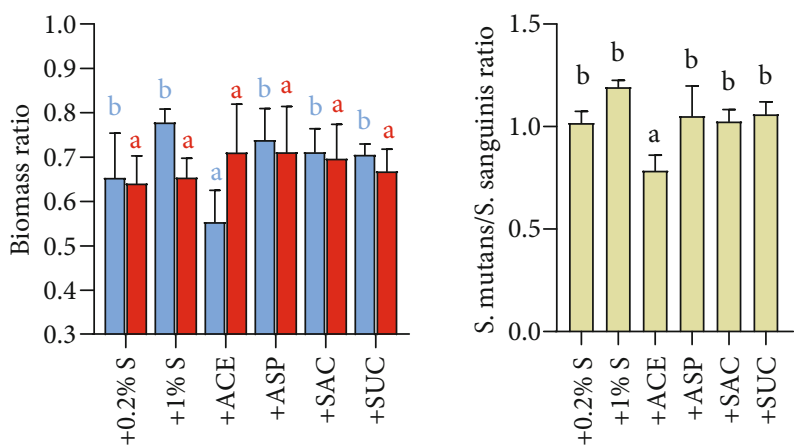

- S. mutans / total bacteria

- S. sanguinis / total bacteria

(b)

Figure 3: Continued. 


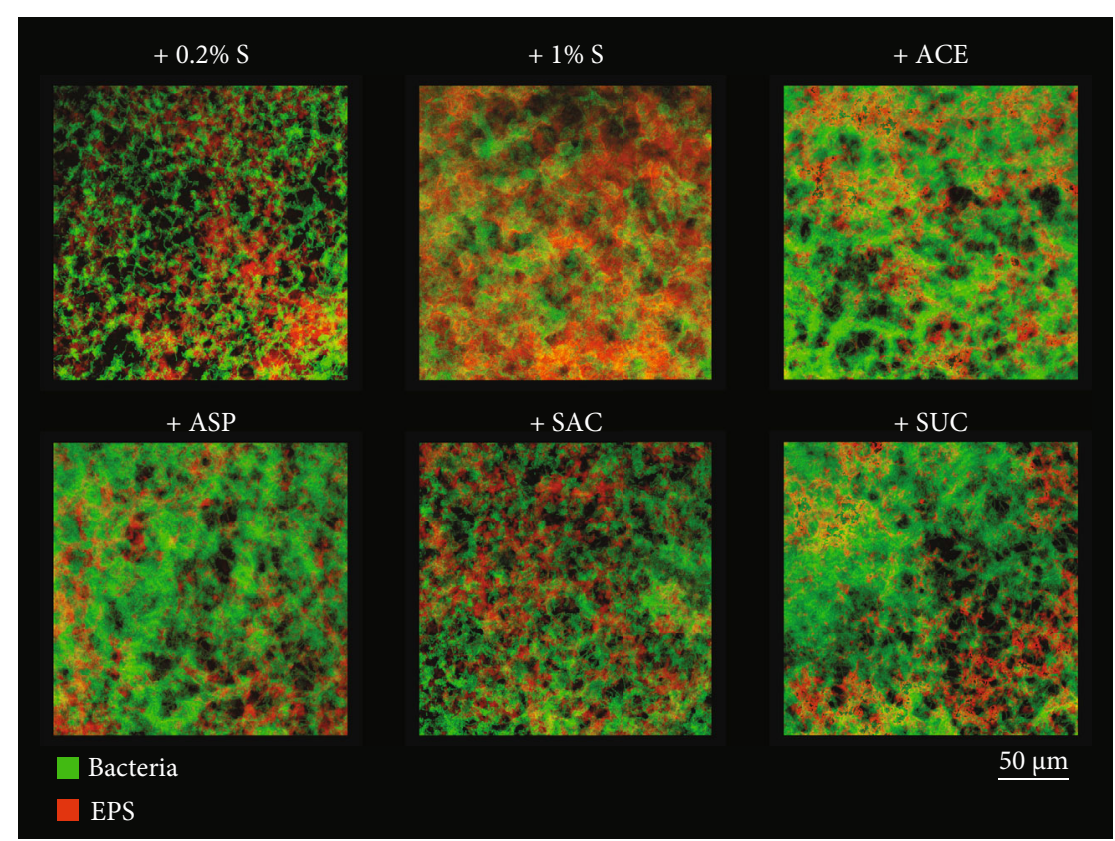

(c)

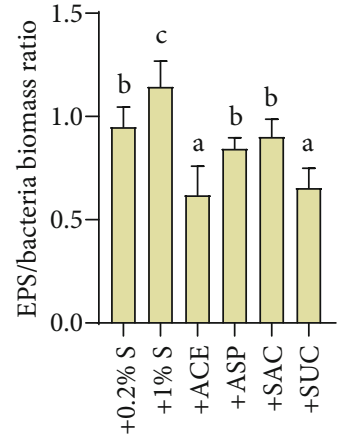

(d)

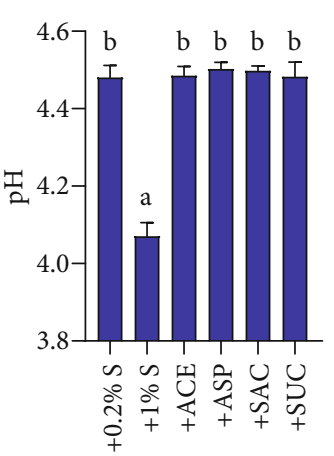

(e)

FIGURE 3: Effects of NNSs on bacterial composition and extracellular polysaccharides (EPS)/acid production of saliva-derived multispecies biofilms. (a) Representative images of multispecies biofilms after fluorescence in situ hybridization. Green: S. mutans; red: S. sanguinis; blue: universal bacteria. (b) Biomass ratio quantitative data of $S$. mutans/universal bacteria (blue), S. sanguinis/universal biomass (red) and S. mutans/S. sanguinis (yellow). (c) Representative images of multispecies biofilms after EPS staining. Green: bacteria; red: EPS. (d) The quantitative data of EPS/bacterial amount in multispecies biofilms. (e) The $\mathrm{pH}$ values of culture media of multispecies biofilms. Results are presented as mean \pm standard deviation. Different lowercase letters indicate a significant intergroup difference and are marked according to mean value. (S: sucrose; ACE: acesulfame-K; ASP: aspartame; SAC: saccharin; SUC: sucralose.).

Despite the fact that the multispecies model derives from human saliva and consists of much more species than selected bacteria [32], the batch culture with constant treatment was insufficient for mimicking the environment of the oral cavity and the daily consumption of NNSs. Moreover, it should be noted that sweeteners are not usually commercialized in their pure form, so the results cannot be directly extended to sugar substitutes in the market when interpreting the data. The stronger evidence for the ecological benefit of NNSs on oral microbiota needed to be provided by welldesigned cohort studies with the subjects sustainably exposed to NNSs. Additionally, some research has reported that certain NNSs induce alterations of the gut microbiome and result in metabolic impairments of the host [33-35]. Therefore, whether NNSs can be metabolized by oral bacteria and the further influence of the interactions on the host are required to be comprehensively explored. As NNSs have diverse chemical structures and properties, it is conceivable that the metabolization of various NNSs has different ecological effects in biofilms. Since some natural extract has been identified as an alternative treatment in caries $[36,37]$, the effect of natural intense sweeteners such as steviol glycosides and thaumatin deserves further exploration.

\section{Conclusion}

Taken together, this study demonstrates that NNSs (acesulfame-K, aspartame, saccharin, and sucralose) suppress the growth and biofilm formation of $S$. mutans or S. sanguinis in planktonic culture and single-species biofilm. These sweeteners decrease $S$. mutans/S. sanguinis ratio and induce less acid/EPS production than sucrose in dual- 
and multispecies biofilm models. The benefit of NNSs for bacterial equilibrium in biofilm may have a promising prospect in caries management.

\section{Data Availability}

All data of the study are available in the article and can be solicited from the corresponding authors.

\section{Conflicts of Interest}

The authors declare no conflict of interest.

\section{Authors' Contributions}

Conceptualization was performed by J. Zhu, J. Liu, X. Zheng, and X. Zhou; methodology was performed by J. Zhu, Z. Li, R. $\mathrm{Xi}$, and Y. Li; formal analysis was performed by J. Zhu, J. Liu, Z. Li, and R. Xi; investigation was performed by J. Zhu, J. Liu, Z. Li, and X. Peng; data curation was performed by Z. Li, Y. $\mathrm{Li}$, and X. Peng. Resources was performed by X. Xu, X. Zheng, and $\mathrm{X}$. Zhou; writing original draft was performed by J. Zhu, J. Liu, and X. Zheng; writing, review, and editing were performed by X. Zheng, X. Xu, and X. Zhou; visualization was performed by J. Zhu and J. Liu; supervision was performed by X. Zheng and X. Zhou; funding acquisition was performed by X. Zheng, X. Xu, and X. Zhou. Jianhui Zhu and Jiaxin Liu contributed equally to this work.

\section{Acknowledgments}

The authors acknowledge helpful discussions from Prof. Robert Margolskee (Monell Chemical Senses Center). This work was supported by the National Natural Science Foundation of China [81900995 to X. Zheng, 81771099 to X. Xu, and 81670978 to X. Zhou], the Postdoctoral Foundation of Sichuan University Grant [2019SCU12021 to X. Zheng], and the Research funding for talents developing, West China Hospital of Stomatology Sichuan University [RCDWJS202011 to $\mathrm{X}$. Zheng].

\section{References}

[1] P. Gupta, N. Gupta, A. P. Pawar, S. S. Birajdar, A. S. Natt, and H. P. Singh, "Role of sugar and sugar substitutes in dental caries: a review," ISRN Dentistry, vol. 2013, Article ID 519421, 2013.

[2] V. S. Malik and F. B. Hu, "Sugar-sweetened beverages and cardiometabolic health: an update of the evidence," Nutrients, vol. 11, no. 8, p. 1840, 2019.

[3] A. C. Sylvetsky, Y. Jin, E. J. Clark, J. A. Welsh, K. I. Rother, and S. A. Talegawkar, "Consumption of low-calorie sweeteners among children and adults in the United States," Journal of the Academy of Nutrition and Dietetics, vol. 117, no. 3, pp. 441-448.e2, 2017.

[4] N. B. Pitts, D. T. Zero, P. D. Marsh et al., "Dental caries," Nature Reviews. Disease Primers, vol. 3, no. 1, article 17030, 2017.

[5] W. H. Bowen and H. Koo, "Biology of Streptococcus mutansderived glucosyltransferases: role in extracellular matrix for- mation of cariogenic biofilms," Caries Research, vol. 45, no. 1, pp. 69-86, 2011.

[6] N. Takahashi and B. Nyvad, "The role of bacteria in the caries process: ecological perspectives," Journal of Dental Research, vol. 90, no. 3, pp. 294-303, 2011.

[7] S. A. Mosaddad, E. Tahmasebi, A. Yazdanian et al., "Oral microbial biofilms: an update," European Journal of Clinical Microbiology \& Infectious Diseases, vol. 38, no. 11, pp. 20052019, 2019.

[8] T. Matsukubo and I. Takazoe, "Sucrose substitutes and their role in caries prevention," International Dental Journal, vol. 56, no. 3, pp. 119-130, 2006.

[9] F. Abdul Razak, B. A. Baharuddin, E. F. M. Akbar, A. H. Norizan, N. F. Ibrahim, and M. Y. Musa, "Alternative sweeteners influence the biomass of oral biofilm," Archives of Oral Biology, vol. 80, pp. 180-184, 2017.

[10] S. C. Durso, L. M. Vieira, J. N. S. Cruz, C. S. Azevedo, P. H. Rodrigues, and M. R. L. Simionato, "Sucrose substitutes affect the cariogenic potential of Streptococcus mutans biofilms," Caries Research, vol. 48, no. 3, pp. 214-222, 2014.

[11] R. A. Giacaman, P. Campos, C. Muñoz-Sandoval, and R. J. Castro, "Cariogenic potential of commercial sweeteners in an experimental biofilm caries model on enamel," Archives of Oral Biology, vol. 58, no. 9, pp. 1116-1122, 2013.

[12] C. H. Edwards, M. Rossi, C. P. Corpe, P. J. Butterworth, and P. R. Ellis, "The role of sugars and sweeteners in food, diet and health: alternatives for the future," Trends in Food Science \& Technology, vol. 56, pp. 158-166, 2016.

[13] K. I. Rother, E. M. Conway, and A. C. Sylvetsky, "How nonnutritive sweeteners influence hormones and health," Trends in Endocrinology and Metabolism: TEM, vol. 29, no. 7, pp. 455-467, 2018.

[14] X. Zheng, X. Cheng, L. Wang et al., "Combinatorial effects of arginine and fluoride on oral bacteria," Journal of Dental Research, vol. 94, no. 2, pp. 344-353, 2015.

[15] L. Guo, J. S. McLean, Y. Yang et al., "Precision-guided antimicrobial peptide as a targeted modulator of human microbial ecology," Proceedings of the National Academy of Sciences of the United States of America, vol. 112, no. 24, pp. 7569-7574, 2015.

[16] X. Zheng, K. Zhang, X. Zhou et al., "Involvement of gshAB in the interspecies competition within oral biofilm," Journal of Dental Research, vol. 92, no. 9, pp. 819-824, 2013.

[17] M. I. Klein, S. Duarte, J. Xiao, S. Mitra, T. H. Foster, and H. Koo, "Structural and molecular basis of the role of starch and sucrose in Streptococcus mutans biofilm development," Applied and Environmental Microbiology, vol. 75, no. 3, pp. 837-841, 2009.

[18] A. Heydorn, A. T. Nielsen, M. Hentzer et al., "Quantification of biofilm structures by the novel computer program COMSTAT," Microbiology, vol. 146, no. 10, pp. 2395-2407, 2000.

[19] W. Ludwig, O. Strunk, R. Westram et al., "ARB: a software environment for sequence data," Nucleic Acids Research, vol. 32, no. 4, pp. 1363-1371, 2004.

[20] P. D. Marsh, "Microbial ecology of dental plaque and its significance in health and disease," Advances in Dental Research, vol. 8, no. 2, pp. 263-271, 1994.

[21] P. D. Marsh, "Are dental diseases examples of ecological catastrophes?," Microbiology, vol. 149, no. 2, pp. 279-294, 2003.

[22] R. J. Lamont, H. Koo, and G. Hajishengallis, "The oral microbiota: dynamic communities and host interactions," Nature Reviews. Microbiology, vol. 16, no. 12, pp. 745-759, 2018. 
[23] I. D. Mandel and V. L. Grotz, "Dental considerations in sucralose use," The Journal of Clinical Dentistry, vol. 13, no. 3, pp. 116-118, 2002.

[24] J. Kreth, Y. Zhang, and M. C. Herzberg, "Streptococcal antagonism in oral biofilms: Streptococcus sanguinis and Streptococcus gordonii interference with Streptococcus mutans," Journal of Bacteriology, vol. 190, no. 13, pp. 4632-4640, 2008.

[25] J. Kreth, J. Merritt, W. Shi, and F. Qi, "Competition and coexistence between Streptococcus mutans and Streptococcus sanguinis in the dental biofilm," Journal of Bacteriology, vol. 187, no. 21, pp. 7193-7203, 2005.

[26] J. A. Banas, "Virulence properties of Streptococcus mutans," Frontiers in Bioscience, vol. 9, no. 1-3, pp. 1267-1277, 2004.

[27] M. I. Klein, G. Hwang, P. H. S. Santos, O. H. Campanella, and H. Koo, "Streptococcus mutans-derived extracellular matrix in cariogenic oral biofilms," Frontiers in Cellular and Infection Microbiology, vol. 5, p. 10, 2015.

[28] H.-C. Flemming and J. Wingender, "The biofilm matrix," Nature Reviews. Microbiology, vol. 8, no. 9, pp. 623-633, 2010.

[29] N. Takahashi, M. Horiuchi, and T. Yamada, "Effects of acidification on growth and glycolysis of Streptococcus sanguis and Streptococcus mutans," Oral Microbiology and Immunology, vol. 12, no. 2, pp. 72-76, 1997.

[30] X. Huang, R. M. Schulte, R. A. Burne, and M. M. Nascimento, "Characterization of the arginolytic microflora provides insights into $\mathrm{pH}$ homeostasis in human oral biofilms," Caries Research, vol. 49, no. 2, pp. 165-176, 2015.

[31] Y.-L. Liu, M. Nascimento, and R. A. Burne, "Progress toward understanding the contribution of alkali generation in dental biofilms to inhibition of dental caries," International Journal of Oral Science, vol. 4, no. 3, pp. 135-140, 2012.

[32] X. Xu, J. He, J. Xue et al., "Oral cavity contains distinct niches with dynamic microbial communities," Environmental Microbiology, vol. 17, no. 3, pp. 699-710, 2015.

[33] J. Suez, T. Korem, D. Zeevi et al., "Artificial sweeteners induce glucose intolerance by altering the gut microbiota," Nature, vol. 514, no. 7521, pp. 181-186, 2014.

[34] J. Suez, T. Korem, G. Zilberman-Schapira, E. Segal, and E. Elinav, "Non-caloric artificial sweeteners and the microbiome: findings and challenges," Gut Microbes, vol. 6, no. 2, pp. 149-155, 2015.

[35] M. B. Abou-Donia, E. M. el-Masry, A. A. Abdel-Rahman, R. E. McLendon, and S. S. Schiffman, "Splenda alters gut microflora and increases intestinal p-glycoprotein and cytochrome p-450 in male rats," Journal of Toxicology and Environmental Health. Part A, vol. 71, no. 21, pp. 1415-1429, 2008.

[36] E. T. Moghadam, M. Yazdanian, E. Tahmasebi et al., "Current herbal medicine as an alternative treatment in dentistry: in vitro, in vivo and clinical studies," European Journal of Pharmacology, vol. 889, p. 173665, 2020.

[37] H. Seifi Kafshgari, M. Yazdanian, R. Ranjbar et al., "The effect of Citrullus colocynthis extracts on Streptococcus mutans, Candida albicans, normal gingival fibroblast and breast cancer cells," Journal of Biological Researchn-Bollettino della Società Italiana di Biologia Sperimentale, vol. 92, no. 1, 2019. 